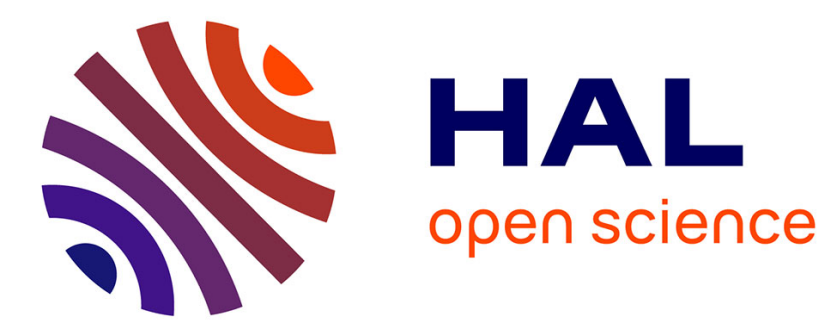

\title{
A new tailored solution to predict blow efficiency and energy consumption of hammer-forging machines
} Jean-François Mull, Camille Durand, Cyrille Baudouin, Régis Bigot

\section{To cite this version:}

Jean-François Mull, Camille Durand, Cyrille Baudouin, Régis Bigot. A new tailored solution to predict blow efficiency and energy consumption of hammer-forging machines. International Journal of Advanced Manufacturing Technology, 2020, 111, pp.1941 - 1954. 10.1007/s00170-020-06237-2 . hal-03047983

\section{HAL Id: hal-03047983 \\ https://hal.science/hal-03047983}

Submitted on 9 Dec 2020

HAL is a multi-disciplinary open access archive for the deposit and dissemination of scientific research documents, whether they are published or not. The documents may come from teaching and research institutions in France or abroad, or from public or private research centers.
L'archive ouverte pluridisciplinaire HAL, est destinée au dépôt et à la diffusion de documents scientifiques de niveau recherche, publiés ou non, émanant des établissements d'enseignement et de recherche français ou étrangers, des laboratoires publics ou privés. 


\title{
A new tailored solution to predict blow efficiency and energy consumption of hammer-forging machines
}

\author{
Jean-François Mull ${ }^{1} \cdot$ Camille Durand $^{1} \cdot$ Cyrille Baudouin $^{1} \cdot$ Régis Bigot $^{1}$
}

\begin{abstract}
Energy saving has become a real issue in process management and has led to the analysis of the energy consumption of forging machines, for the purpose of optimization. This study focuses on the amount of energy lost due to machine behaviour during the forming process. A spring-mass-damping model of the machine and tools system is associated with a billet model to describe hammer-forming operations only during the forging phase. The model parameters are identified with experimental measurements of process variables during a stroke, providing parameters specifically adapted to the machine-tools system. Then, model predictions are validated by the experimental upsetting of steel and aluminium billets. The model accurately predicts forging process variables for consecutive blows with different materials. The decrease in process efficiency and the evolution from inelastic to elastic blows after several strokes on the same billet are also predicted by the model. This methodology provides a new, tailored solution that enables forging manufacturers to predict the forging energy consumption of their own machines. The approach developed in this work concerns gravity drop hammers but is also transferable to other energy-driven machines.
\end{abstract}

\section{Introduction}

Improvements in the understanding of material flow behaviour and phenomena at the billet-tools interface imply that other elements, such as machine behaviour, can no longer be neglected in simulations. Despite good characterisation of material and interface behaviours, Durand et al. [1] have shown that simulations do not provide a representative picture of the situation for high-performing material, forged on energydriven machines. For sheet metal forming applications, Bigot et al. [2] highlight that the machine-tools deflection

\author{
Jean-François \\ Mulljeanfrancois.mull@ens \\ am.eu \\ Camille Durand \\ camille.durand@ensam.eu \\ Cyrille Baudouin \\ cyrille.baudouin@ensam.eu \\ Régis Bigot \\ regis.bigot@ensam.eu \\ 1 Arts et Metiers Institute of Technology, Université de Lorraine, \\ LCFC, HESAM Université, F-57070 Metz, France
}

caused by the reaction forces from the workpiece affects the tolerances of the components, showing the impact of the machine behaviour on the forging process. Brecher et al. [3] consider the simple representation of the machine in forging process simulations to be one of the main reasons for the lack of accuracy. Thus, better consideration of machine behaviour could improve the prediction of energy consumption and forging variables, such as load or ram displacement.

Gravity-drop hammers are forging machines, which raise a ram to a given height before dropping it. Gravity then accelerates the ram until it reaches the billet to deform it. During forging operations, especially with hammers, extreme conditions can be noticed concerning temperature, impact velocity and developed energy. This implies that the instrumentation of forging machines is difficult, which is why theoretical models are used to describe dynamic machine behaviour. Tobias [4] developed a one degree of freedom model with one mass and one spring to represent hammer behaviour and Vajpayee et al. [5] proposed a model comprising one spring and two masses to describe hammer-forging operations. The masses and the stiffness were obtained from the machine specifications and the machine model was coupled with the billet behaviour. In another study, Vajpayee et al. [6] refined the model with a damper linked to the spring with a parallel connection. 
These models were used to observe the influence of process parameters on the process efficiency. Gregorian et al. [7] applied a similar approach, with the aim of predicting the sound emitted by machine vibrations for a laboratory drop hammer and Vajpayee et al. [8] studied the noise emitted by a highenergy-rate-forming machine. Several models which were dependent on the foundation design were proposed and focused on the response of the foundation under hammer solicitations. Vajpayee and Sadek [9] developed a model with three masses to consider the foundation behaviour. Novak [10] calculated the parameters associated with a two degrees of freedom mod$\mathrm{el}$, according to theoretical methods and assumptions on the materials and geometries of foundation elements. Novak and El Hifnawy [11] developed a complex eigenvalue method to study hammers comprising an eccentrically mounted anvil. Thanks to the development of these models, El Hifnawy and Novak [12] studied the response of hammer foundations for different pulses loading and Harwood and Novak [13] investigated the uplift phenomenon in hammer foundations. Chow and Yong [14] developed a one-dimensional wave equation model based on the finite element method. Model predictions were compared to experimental measurements as well as results obtained by Harwood and Novak [13] with a springmass-damping model. Chehab and El Naggar [15] completed the foundation model for hammers, with the consideration of a base isolator. By studying the impact of the isolator model parameters on the maximum blow force, the authors were able to provide a decision-making tool which permits the choice of a mounting system depending on the application. In simulation, Chehab and El Naggar [16] investigated the effect of pulse shape and pulse duration on the dynamic response of the one mass hammer foundation system. Wang and Dong [17] optimised model parameters of an isolator represented by a two degree of freedom system to minimise maximum impact force. Furthermore, Heidari and El Naggar [18] studied on the influence of reinforced soil systems on vibration propagation. More recently, Dindorf and Wos [19] developed an energy-efficient power supply and an intelligent dynamic control system for a hydraulic forging press with the purpose of saving energy and resources. Jomartov et al. [20] focused on the dynamics of the brake of a crank press. The driving system was modelled in the SimulationX software and allowed to predict ram, drive shaft and crank shaft vibrations during the braking phase. Moreover, finite-element simulations were used to provide more sophisticated models of machine-tools systems. Swidergal et al. [21] developed a model coupling a multi-body system with the finite element method (MBSFEM) for sheet metal forming applications. The MBS model of a mechanical press was established, with a 3D CAD of the structure. Then, the behaviour of the machine was coupled with the FE simulation of the sheet upsetting. In the case of a mechanical press, Zheng and Zhou [22] proposed a hybrid model considering the elastic behaviour of the crank shaft, simulated by FE simulation, resulting in a rigid-flexible coupling model of the slider-crank mechanism. Brecher et al. [23] proposed a similar approach in the case of closed die forging process, the machine and tools system was modelled with a MBS and billet behaviour was described with a FE model. These methodologies are adequate approaches to reduce computation time compared to a FE simulation of the whole system (machine + material). Nevertheless, it requires accurate knowledge about the machine structure and the forging operation, to provide accurate simulations. As can be seen, dynamic models were proposed to represent the dynamic behaviour of forging machines but the difficulties in measuring process variables during the forming process, implied that no experimental methods were proposed to identify model parameters.

Concerning the hammer-forging machines of interest in this paper, recent studies of the process efficiency were performed but they only considered the energy consumption before the impact with the billet. Hongtang and Bufang [24] assessed the efficiency of ram-driven systems for a steamdriven hammer and an electro-hydraulic hammer. The study highlighted that electro-hydraulic hammers are relevant to energy consumption reduction. Zhang [25] developed a mathematical model for the whole hydraulic-driven system, to improve process control and enhance the working efficiency of hammers. Zhu et al. [26] proposed a new design of the electromagnetic driver for reversing valves to improve blow energy control and driver mechanism robustness. Hammer instrumentation with modern measurement systems allowed deeper investigation during the forming operations. With the help of a high-speed camera, Galdos et al. [27] determined the impact velocity for copper upsetting on a counterblow hammer. The kinetic energy input from the rams and plastic energy transmitted to the billet were compared, showing a good agreement if blow efficiency was considered. Chen et al. [28] realised similar measurements for a counterblow hammer with a stereo-vision system comprising two high-speed cameras. Furthermore, Yoneyama [29] developed an adapted gravitydrop hammer device to measure load, pressure, friction, and contact temperature. Yoneyama et al. [30] used the device for experimental investigations of the frictional effects during the upsetting of cylindrical steel billets. These new measurement methods allow the observation of phenomena that occur during the forming process. It has become possible to study energy consumption during billet forming. Thus, models describing the forming operation, with consideration of machine and tools behaviour, can be developed from experimental observations.

In this paper, an experimental methodology is developed with the aim of determining the best-suited spring-massdamping system to model forming operations on a gravitydrop hammer. Measurements of the process variables during billet forming for reference blows allow us to obtain model parameters specifically adapted to the machine-tools system. 
The main contribution of this paper is to provide a predictive tool to calculate the blow efficiency of hammer operations and enhance the understanding of energy consumption in forging processes. The methodology that allows to model forming operations and identifies the associated parameters is given in section 2. Section 3 focuses on the validation of the model by comparing experiments and simulations for two series of blows on steel and aluminium billets. The discussion and conclusions are presented in sections 4 and 5 , respectively.

\section{Identification of the model and its parameters}

\subsection{Experimental protocol}

Billet forming is performed with the MONTBARD® LG1000 gravity-drop hammer from the lycée Marie Curie in Nogentsur-Oise, France. The upper and lower tools are both flat anvils. The mass of the ram-upper tool system is estimated to be $1250 \mathrm{~kg}$. Under the lower tool, a KISTLER ${ }^{\circledR}$ KI9241B14 piezoelectric transversal sensor is fixed, to allow the measurement of the forging load during the billet upsetting. For the recording of the load signal, an analogue data acquisition module (EUROSMART® SYSAM-SP5) and the EUROSMART® LATIS-Pro software are used. The motion of the ram is recorded with a LUMIX® FZ200 camera. With this recording and the AVIMECA $3 \AA$ software, the displacement of the ram is obtained. The derivative of the displacement is then calculated and the impact velocity determined; it is defined as the velocity of the ram when the upper tool reaches the upper surface of the billet. A global view of the machine, the tools and the sensor used for the experimental protocol is presented in Fig. 1.

The protocol consists of the upsetting of a $16 \mathrm{NiCrMo} 13$ steel cylinder of $60 \mathrm{~mm}$ diameter and $112 \mathrm{~mm}$ height. The billet is heated to reach $1100^{\circ} \mathrm{C}$ and is lubricated with a mix of oil and graphite before the first stroke. Seven consecutive blows are performed on the same billet.

To estimate the billet height after each blow, six control billets were upset and measured: for the determination of the billet height of the $i$ th blow, $i$ blows are performed on one control billet, the forging operation is stopped and the billet is then measured.

In section 2 , only the load signal of the 7 th blow is exploited for the identification of the model parameters (Fig. 2).

\subsection{Spectral analysis}

Spectral analysis is the step that allows us to determine the number of degrees of freedom of the system. A fast Fourier transform (FFT) is performed on the load signal coming from the piezoelectric sensor. Before applying the FFT, a post-treatment of the load signal is required. Indeed, the resolution of the FFT spectrum is calculated according to the length time of the signal $\left(t_{\text {exp }}\right)$ and is equal to $R=1 / t_{\text {exp }}$. In this case, the resolution is too high to identify the vibratory modes that make up the signal. Therefore, the zero-padding method is used to decrease the frequency distance between bins in the FFT [31]. The method consists of adding zeroes at the end of the time samples. The consequence is a lengthening of the signal sequence, which decreases the apparent resolution of the FFT. MATLAB ${ }^{\circledR}$ is then used to perform the FFT with the ' $\mathrm{fft}$ ' function. The spectrum is presented in Fig. 3.

Two peaks can be identified with the frequency spectrum. The first, and most important, is the peak for the frequency 0 $\mathrm{Hz}$, corresponding to the carrier wave. This peak can be explained by a vibratory mode in which the oscillation frequency is lower than the resolution of the FFT. Otherwise, a load variation without vibration, which would result for the FFT in a signal with a frequency that is infinitely small, compared to the resolution of the spectrum. In both cases, this indicates an additional degree of freedom in the system, which must be considered. The second peak, associated with a frequency of about $1.8 \mathrm{kHz}$, corresponds to the vibrations oscillating on the carrier wave (Fig. 2). Even if the characteristics of the carrier wave cannot be identified, the FFT highlights the existence of two degrees of freedom in the system.

\subsection{Modelling}

\subsubsection{Billet-interface-machine (BIM) model}

According to the results of the spectral analysis associated with the load signal, a two degrees of freedom, spring-massdamping vibration model is proposed to describe the machinetools' behaviour during forging operations (Fig. 4). This model is constituted of two masses, one damper and one spring. The two masses represent the parts in motion in the system; $m_{l}$ represents the ram and the upper tool, whereas $m_{2}$ represent the parts in motion in the lower tool-sensor system. The spring $k$ and the damper $c$ link mass 2 to the frame and model the elastic deformations and the damping effects in the structure, respectively. Furthermore, the billet is in contact with the upper and lower tool during the forging process, which implies the application of the forging load $F$ on the masses 1 and 2 .

The application of the fundamental principle of dynamics to mass 1 and to mass 2 provide the dynamic equations of the system, as presented in equations (1) and (2).

$$
\begin{aligned}
& m_{1} \ddot{X}_{1}=F \\
& m_{2} \ddot{X}_{2}=-k X_{2}-c \dot{X}_{2}-F
\end{aligned}
$$


Fig. 1 a Global view of the machine. $\mathbf{b}$ Tools and sensor used for the experimental protocol
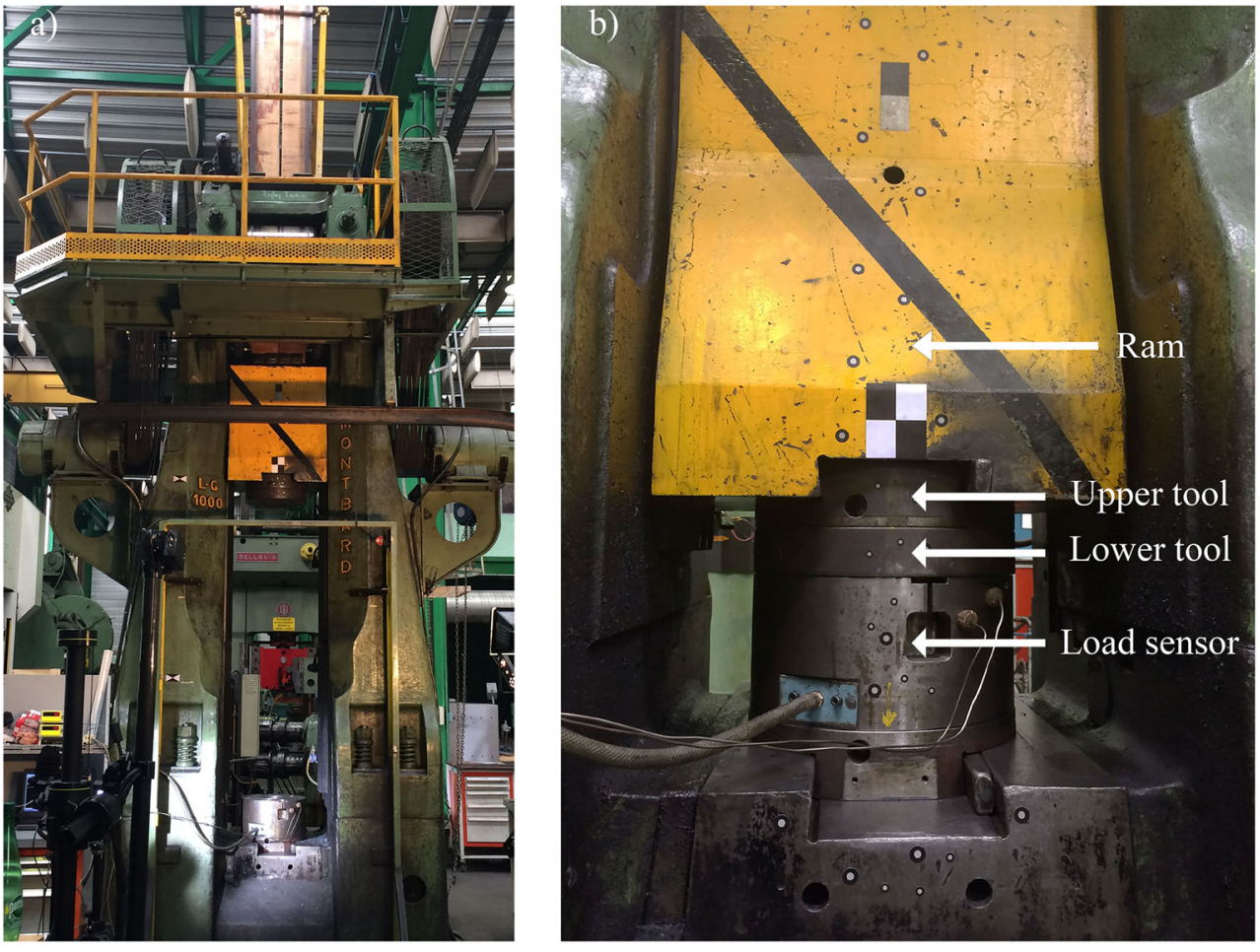

where $X_{1}$ is the displacement of mass 1 and $X_{2}$ is the displacement of mass 2 , according to their position at $t=0$. The velocity $v_{0}$, corresponding to the experimental impact velocity of the ram, is assigned to mass 1 as well as mass 2 .

Clearances were noticed in the lower tool-sensor system. Thus, when mass 1 impacts the billet, billet forming does not start directly because of the clearances catching up. During this phase of clearance catch-up, it is assumed that mass 2 is accelerated to the velocity $v_{0}$.

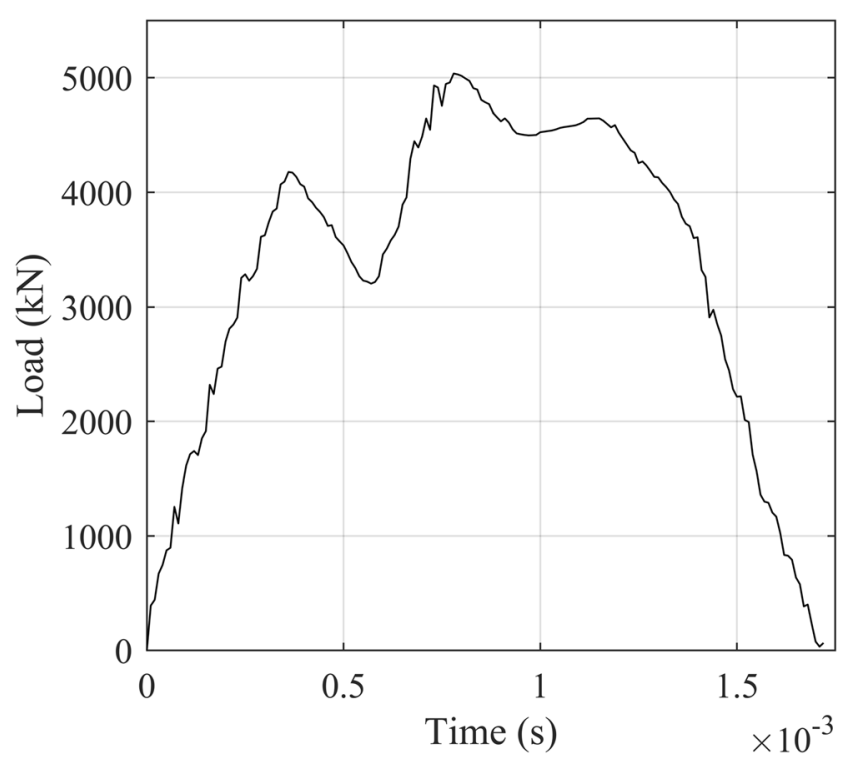

Fig. 2 Recoding of the load signal for the 7th blow on the steel billet

\subsubsection{Modelling the billet behaviour}

For cylinder upsetting, the forging load can be obtained either by numerical or analytical methods. In this study, the load is calculated analytically [32] in order to implement the springmass-damping model and the billet model in one software. The load imposed by the billet is obtained according to equation (3).

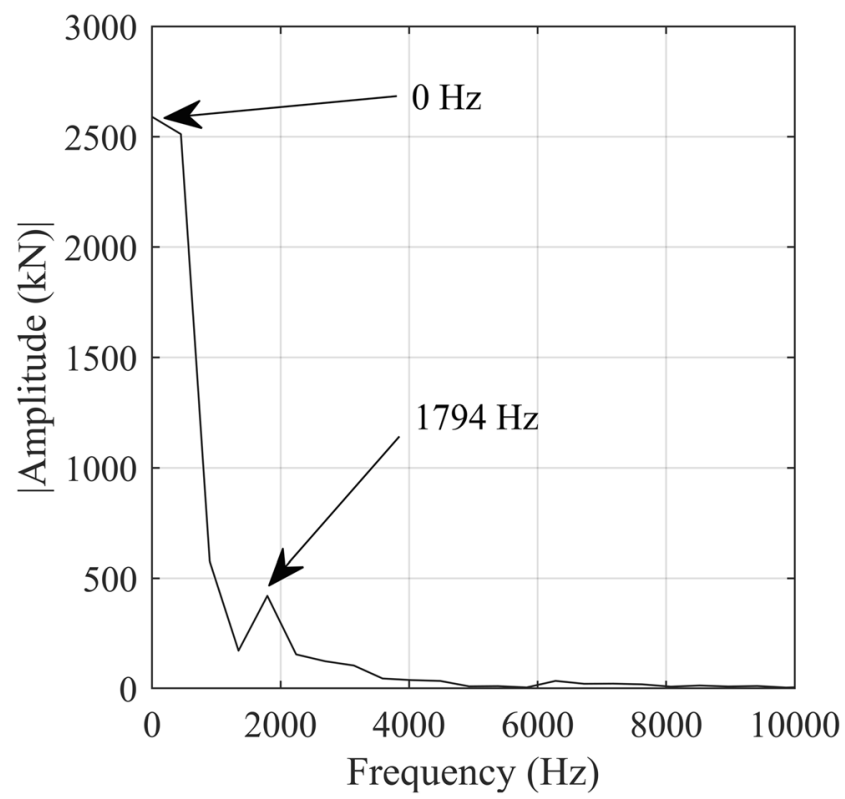

Fig. 3 Frequency spectrum of the load signal for the 7 th blow on the steel billet 


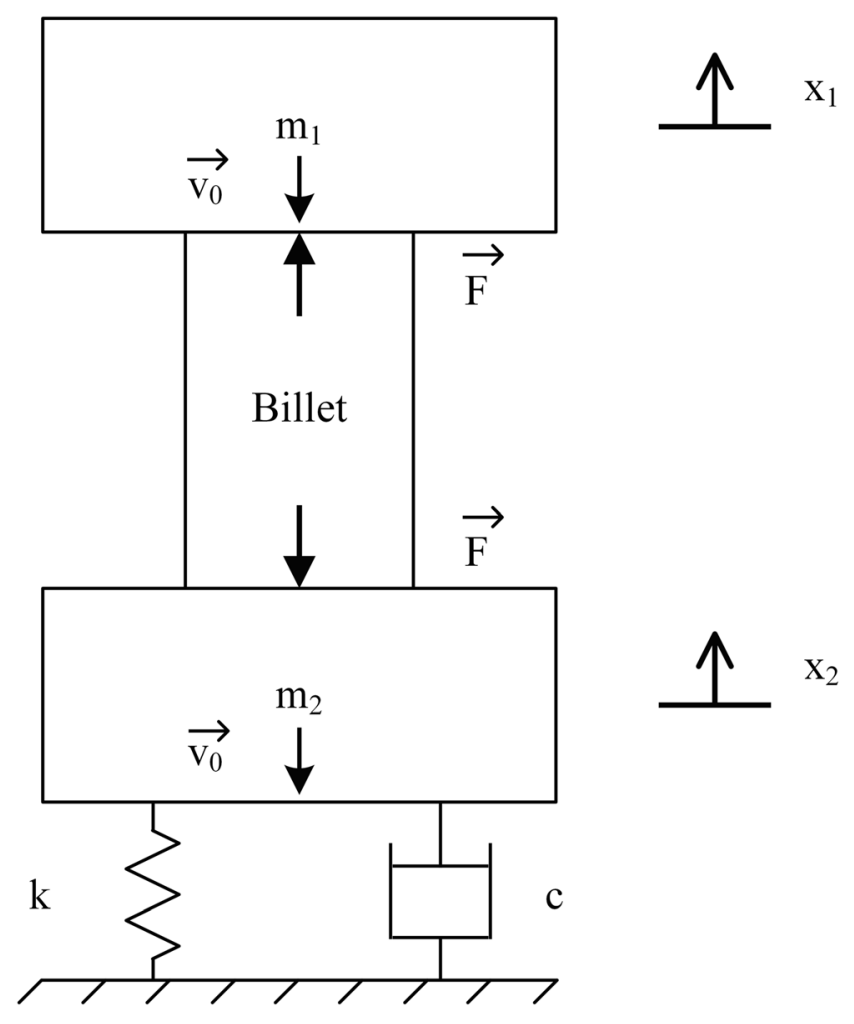

Fig. 4 Billet-interface-machine (BIM) model for the MONTBARD ${ }^{\circledR}$ LG1000 hammer

$F=\frac{\pi r_{e} h}{\mu} \sigma_{0}\left(\frac{e^{A}-1}{A}-1\right)$

where $r_{e}$ is the current radius of the billet, $h$ the current height of the billet, $\mu$ the Coulomb friction coefficient, $\sigma_{0}$ the stress of the material and $A=2 \mu r_{e} / h$.

It is assumed that the specimen keeps its cylindrical form and its initial volume during the deformation. This expression allows consideration of the friction phenomena at the interface of the tools and billet. According to equation (3), $\sigma_{0}$ and $\mu$ need to be calculated in order to find the forging load.

In order to determine the Coulomb friction coefficient $\mu$, test ring compressions are performed. This method is commonly used because of the high sensitivity of the ring geometries to friction throughout the compression [33]. Two steel rings are upset and they are machined to obtain an outer diameter of $120 \mathrm{~mm}$, an inner diameter of $60 \mathrm{~mm}$ and a height of $20 \mathrm{~mm}$. Rings are heated to $1100{ }^{\circ} \mathrm{C}$ and lubricated before the stroke, with a mix of oil and graphite. One blow is performed with the hammer for the first ring, whereas two blows are performed for the second ring. Measurements of radius variation and deformations after the blows are reported on an abacus and a mean friction coefficient is estimated at $\mu=0.2$.

Furthermore, the flow stress is considered constant and is determined by experiment. With equation (3), it is possible to link the billet height to the load applied by the billet to the machine. Equation (3) is solved in $\sigma_{0}$, to obtain the final blow force for the final billet height, both being measured during the experiment. This method provides a flow stress equal to 90 MPa.

The model is called the "Billet-Interface-Machine" (BIM) model because it allows the coupling of these three aspects in simulation. The billet behaviour and the impact of friction at the interface between material and tools are both considered with the expression of the forging load (equation (3)). Furthermore, the machine tools' behaviour is described by the spring-mass-damping model by considering damping effects and elastic strains in the system. The model is implemented on MATLAB SIMULINK $®$ and model equations are solved with the fixed-step solver ode8 (Dormand-Prince method). The step size is fixed to $10^{-7} \mathrm{~s}$. At the beginning of the simulation, mass 1 is in contact with the billet and the initial velocity of both masses is fixed to $-5.3 \mathrm{~m} / \mathrm{s}$, as in the experimental conditions. The model parameters $m 1, m 2, c$ and $k$ are identified by fitting the simulated load signal to the measured load signal.

\subsection{Accuracy of the model}

Figure 5 shows a comparison of the experimental load signal with the simulated load signal obtained with the BIM model and the identified parameters. As can be seen, the simulated load only presents a slight deviation compared to the experimental signal. The maximal simulated load is equal to 4772 $\mathrm{kN}$ whereas the maximal experimental load is equal to 5028 $\mathrm{kN}$ : meaning a relative deviation of $5 \%$. Concerning forging

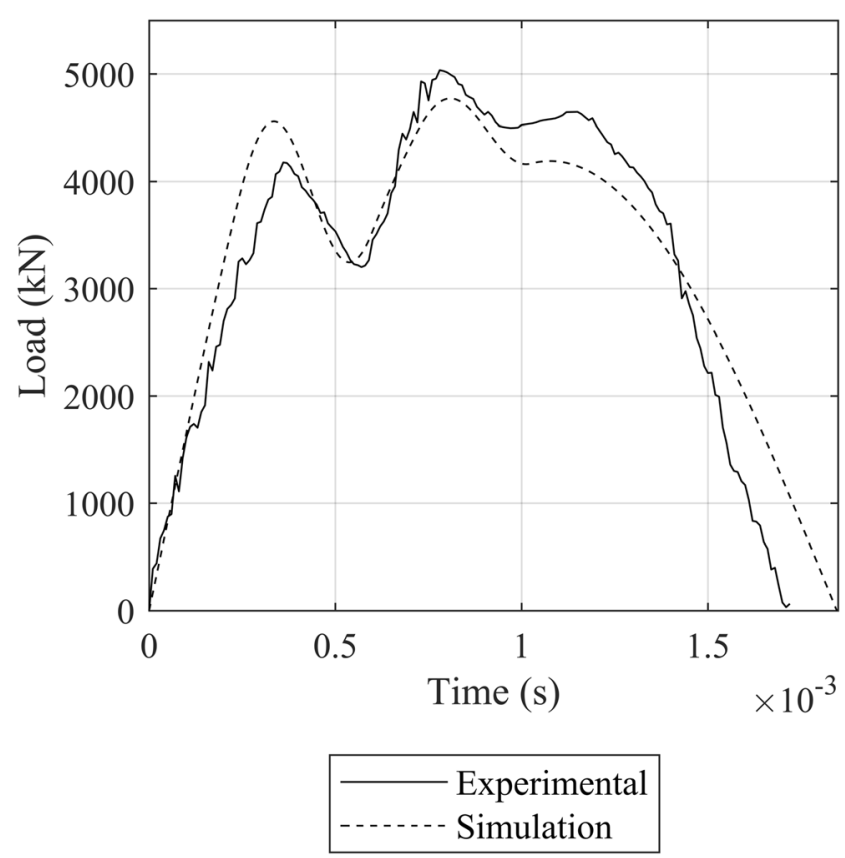

Fig. 5 Experimental load signal and fitted load signal with the BIM model for the 7 th stroke on the steel billet 
times, they are $1.8 \mathrm{~ms}$ and $1.7 \mathrm{~ms}$ in simulation and in experiment, respectively. This corresponds to a relative deviation of $6 \%$.

The parameters identified for the model are presented in Table 1. Mass 1 corresponds to the system (ram + upper tool). According to the experimental assembly, the mass of the ram is equal to $1250 \mathrm{~kg}$. Thus, the identified mass is $41 \%$ lower than the expected value. According to the measurement of the maximal impact velocity $(5.7 \mathrm{~m} / \mathrm{s})$ obtained with another blow, it is possible to calculate the maximal stroke energy as being equal to $12 \mathrm{~kJ}$. The theoretical maximal stroke energy of the hammer is $20 \mathrm{~kJ}$, meaning a relative deviation equal to $39 \%$.

\subsection{Precision of the model}

The Monte-Carlo method [34] is used to assess the impact of process parameter uncertainties on model predictions. Uncertainties associated with measurements of impact velocity, friction coefficient and load are estimated (Table 2). The process parameters are supposed to be random variables following normal laws. Standard deviations are calculated by assuming that uncertainties represent the values, which can be taken by random variables with a confidence level of $95 \%$.

According to the normal laws associated with process parameters, 3000 sets of process parameters are generated. Simulations are then performed for each of these sets, with the BIM model and parameters identified in the previous section (Table 1). Figure 6 shows that the mean of the maximum load and the minimum displacement of mass 1 display asymptotic behaviour when the number of iterations of the MonteCarlo method increases. In addition, load and displacement variations are less than $0.01 \%$ between iteration 2000 and 3000. Therefore, 3000 iterations seem sufficient to ensure the convergence of the Monte-Carlo method.

With results from the 3000 simulations, the bounds of the simulated load signal for a confidence level of $95 \%$, considering process parameter uncertainties, can be determined, as presented in Fig. 7.

At the interval $[0.18 ; 0.33] \mathrm{ms}$, differences between experimental and simulated load are low for a short time period, compared to the forging time. After $t=1.5 \mathrm{~ms}$, larger deviations occur. This shows that the model cannot represent the

Table 1 BIM model parameters identified with the 7th blow on the steel billet

\begin{tabular}{ll}
\hline Parameter & Identified value \\
\hline $\mathrm{m}_{1}(\mathrm{~kg})$ & 732 \\
$\mathrm{~m}_{2}(\mathrm{~kg})$ & 19 \\
$\mathrm{c}(\mathrm{N} . \mathrm{s} / \mathrm{m})$ & 18540 \\
$\mathrm{k}(\mathrm{N} / \mathrm{m})$ & $3.15 \cdot 10^{9}$ \\
\hline
\end{tabular}

Table 2 Uncertainty associated with process parameters and load

\begin{tabular}{lll}
\hline Variable & Uncertainty & Standard deviation \\
\hline Impact velocity & $\pm 0.20 \mathrm{~m} / \mathrm{s}$ & 0.10 \\
Friction coefficient & \pm 0.05 & 0.025 \\
Load & $\pm 300 \mathrm{kN}$ & 150 \\
\hline
\end{tabular}

spring back when the elastic deformations of the structure are relaxed.

\section{Predictivity of the model}

Sections 3.1 and 3.2 present two validation protocols for the BIM model developed in section 2. Process variables as well as billet material are changed to evaluate the range of conditions over which the model is a useful predictor of process behaviour. Furthermore, energy distribution is analysed and compared to results in the literature, to complete the understanding of energy consumption during the forming process.

\subsection{Prediction of the first and the third blow for the upsetting of the steel billet}

\subsubsection{Experimental protocol}

The experimental protocol is the same as presented in section 2.1 but, in this case, the first and the third blows are studied. Note that these blows were not used for the parameter identification.
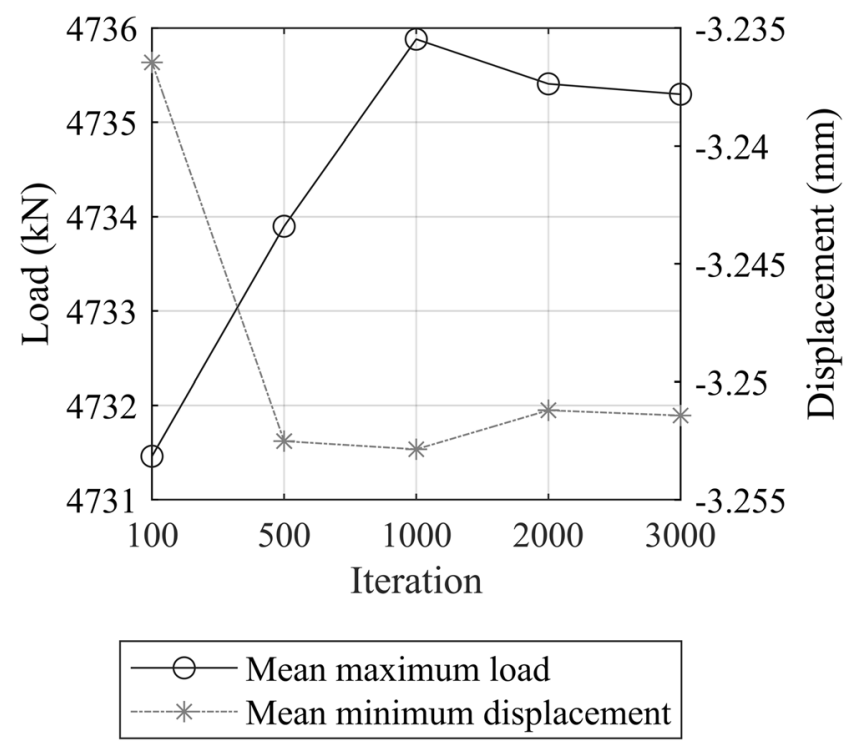

Fig. 6 Mean of the maximum load and the minimum displacement of mass $m_{1}$ for different iterations of the Monte-Carlo method 


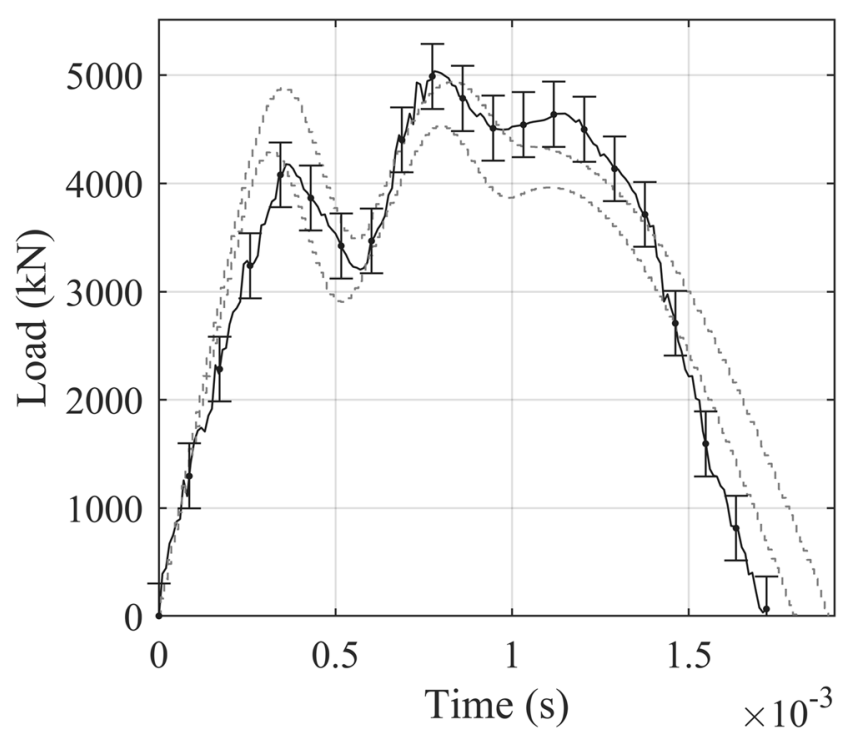

\begin{tabular}{|l|}
\hline Experimental \\
\hline- Upper lower bounds simulation
\end{tabular}

Fig. 7 Experimental load and bounds of the simulated load considering uncertainties of process parameters

\subsubsection{Simulation}

The model used to simulate the first and the third blow is the same as that presented in section 2.3.1. The model parameters used correspond to the parameters identified from Table 1 . The friction coefficient is fixed to the previously determined value. Concerning the flow stress, it is fixed to the value identified in section 2.3.2.

The initial billet height used in the simulation is obtained with the method described in section 2.1. Impact velocities for the first and the third blows are also measured with the camera and were found to be $3.9 \mathrm{~m} / \mathrm{s}$ and $5.3 \mathrm{~m} / \mathrm{s}$, respectively.

\subsubsection{Results}

Several indicators are determined to compare the simulation results with experimental measurements. Because of the vibrations of the second vibratory mode during the forging process, the maximum load measured by the sensor does not occur at the same time as the end of the billet forming. Thus, in order to study the following signals, the final load is introduced and defined as the load when the billet reaches its minimum height. The time to reach the final load is defined as the forging time. At this moment, the final billet height is reached which corresponds to the minimum of the billet height. The time between the instant of the final loading until the load returning to zero is defined as the spring back time.

Figure 8 shows the measured load and the simulated load for the spring $k$ in the case of the first blow. Table 3 presents the indicators studied to compare the simulation to the

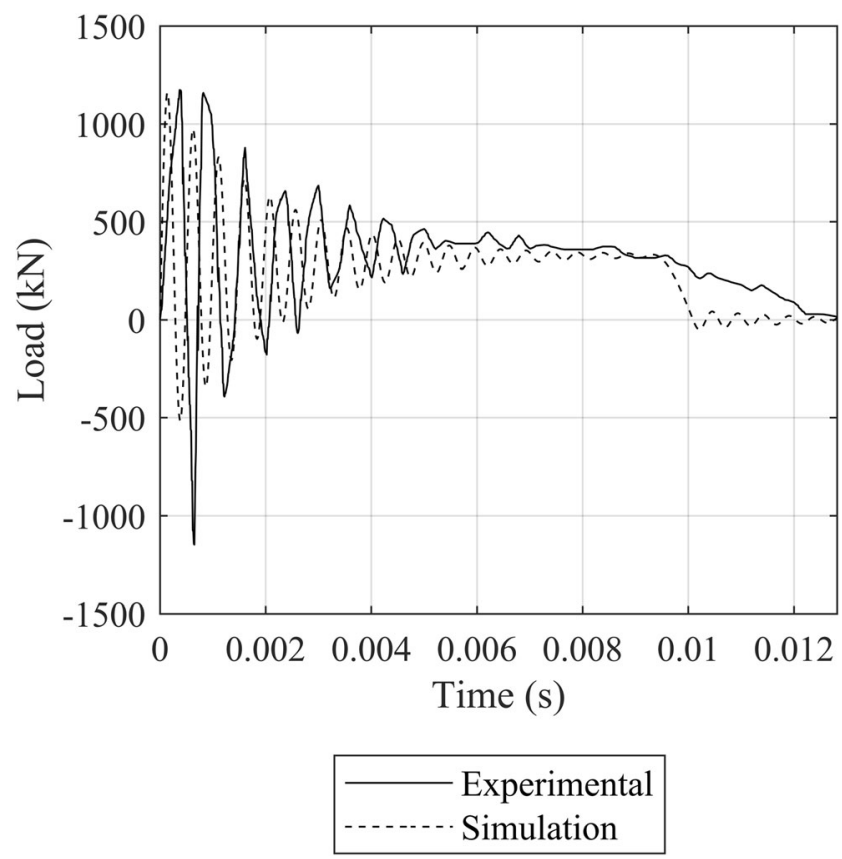

Fig. 8 Measured and simulated load for the 1st blow on the steel billet

experiment. Until the final load is reached, the simulated and the experimental load signals are similar. Simulated indicators present slight deviations from the experiment. During the spring back, bigger differences are observed between experimental and simulated signals. As can be seen, the experimental spring back time is 4.6 times higher than in the simulation.

Concerning the second vibratory mode, the frequency spectrums bring additional information (Fig. 9). The frequency of the experimental and simulated signal presents a relative deviation of $39 \%$ whereas the amplitude of the experimental signal is a little higher, with a relative deviation equal to $38 \%$. The FFT indicates a slight difference in the vibration mode frequency and amplitude, which shows that the second vibratory mode vibrations are well predicted.

Experimental and simulated load signals for the 3rd blow are presented in Fig. 10, and Table 4 presents the indicators for the third blow. As well as for the 1st blow, both signals show the same tendencies before reaching the final load, after this, the model does not provide a good representation of the spring back.

Table 3 Results from the experiment and simulation for the first blow on the steel billet

\begin{tabular}{llll}
\hline & Experiment & Simulation & Relative deviation \\
\hline Final load $(\mathrm{kN})$ & 374 & 333 & $11 \%$ \\
Forming time $(\mathrm{ms})$ & 8.6 & 9.3 & $8 \%$ \\
Final billet height $(\mathrm{mm})$ & 89 & 93 & $4 \%$ \\
Spring back time $(\mathrm{ms})$ & 3.7 & 0.8 & $78 \%$ \\
\hline
\end{tabular}



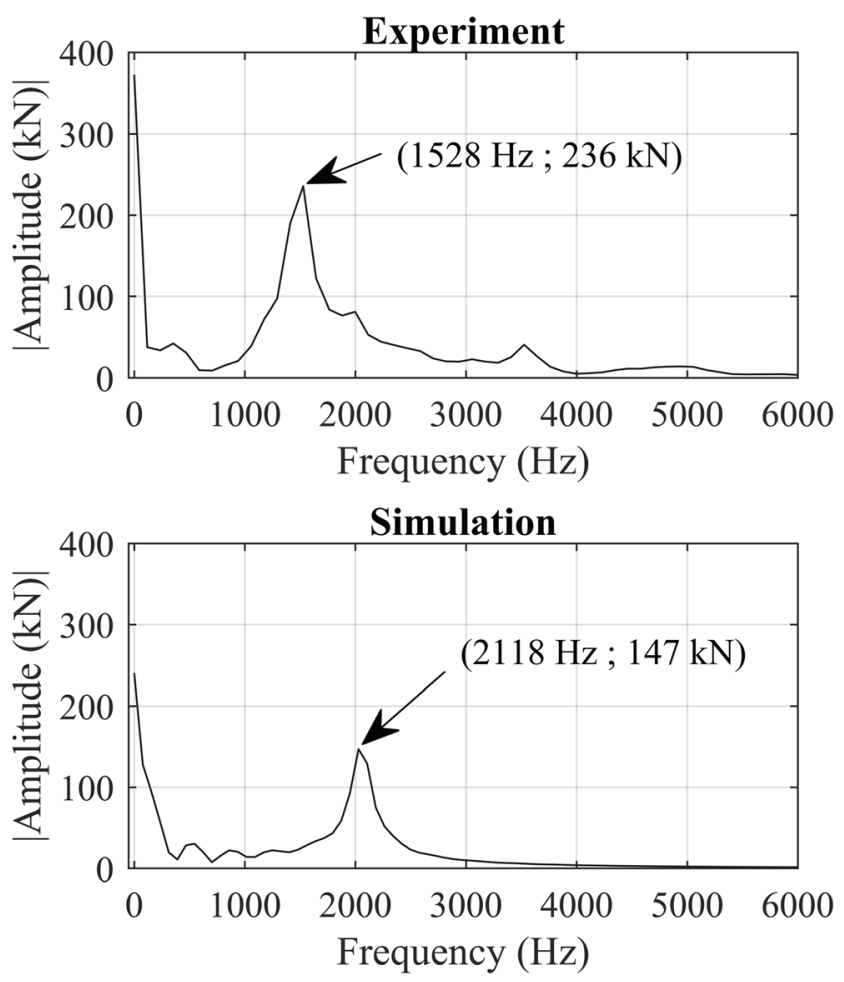

Fig. 9 Frequency spectrums of the experimental and simulated load signals for the 1st blow on the steel billet

The frequency spectrums of the experimental and simulated load signals are shown in Fig. 11. It can be seen that the frequency of the experimental signal is very close to the frequency of the simulated signal with a relative deviation

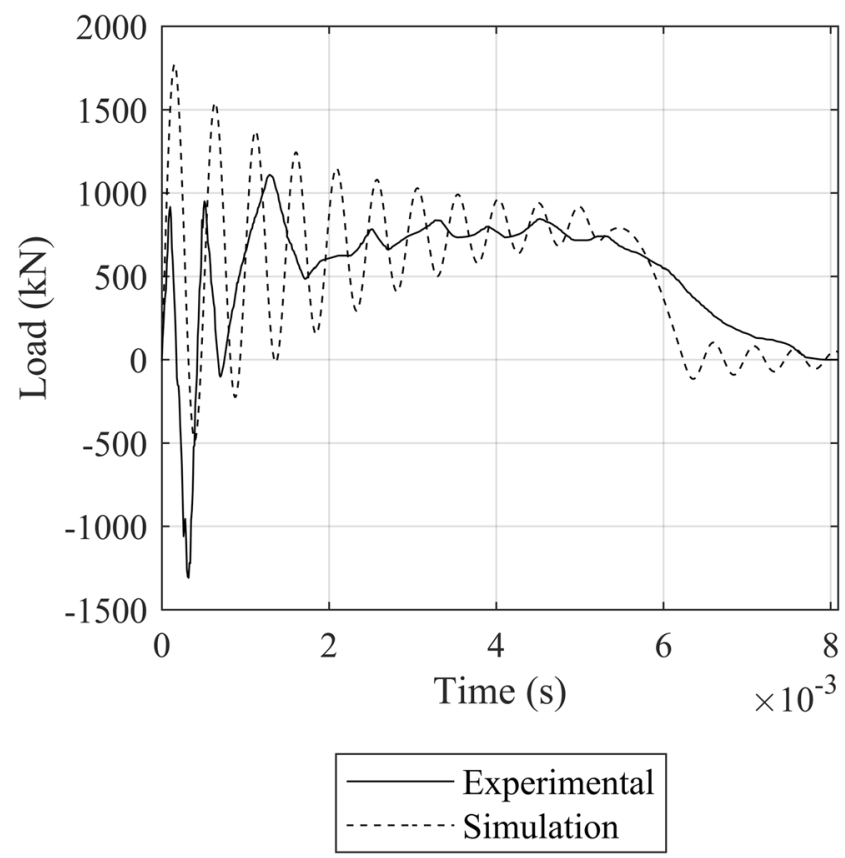

Fig. 10 Measured and simulated load for the 3rd blow on the steel billet
Table 4 Results from experiment and simulation for the third blow on the steel billet

\begin{tabular}{llll}
\hline & Experiment & Simulation & Relative deviation \\
\hline Final load $(\mathrm{kN})$ & 843 & 789 & $6 \%$ \\
Forming time $(\mathrm{ms})$ & 4.5 & 5.5 & $22 \%$ \\
Final billet height $(\mathrm{mm})$ & 37 & 42 & $14 \%$ \\
Spring back time $(\mathrm{ms})$ & 3.2 & 0.8 & $75 \%$ \\
\hline
\end{tabular}

of $1.5 \%$. Concerning the amplitude of the signal, bigger differences can be seen. The amplitude of the simulated vibrations is $72 \%$ higher than the amplitude of the experimental signal.

\subsection{Prediction of consecutive blows for the upsetting of an aluminium billet}

\subsubsection{Experimental protocol}

The upsetting of a 7175-aluminium cylinder of $60 \mathrm{~mm}$ diameter and $112 \mathrm{~mm}$ height is performed to validate the prediction of the model for another material. Six blows are realised, with the same machine and tools used for parameter identification. Experimental measurements are realised in the same conditions presented in section 2.1 and a friction coefficient of 0.07 is identified for the aluminium.
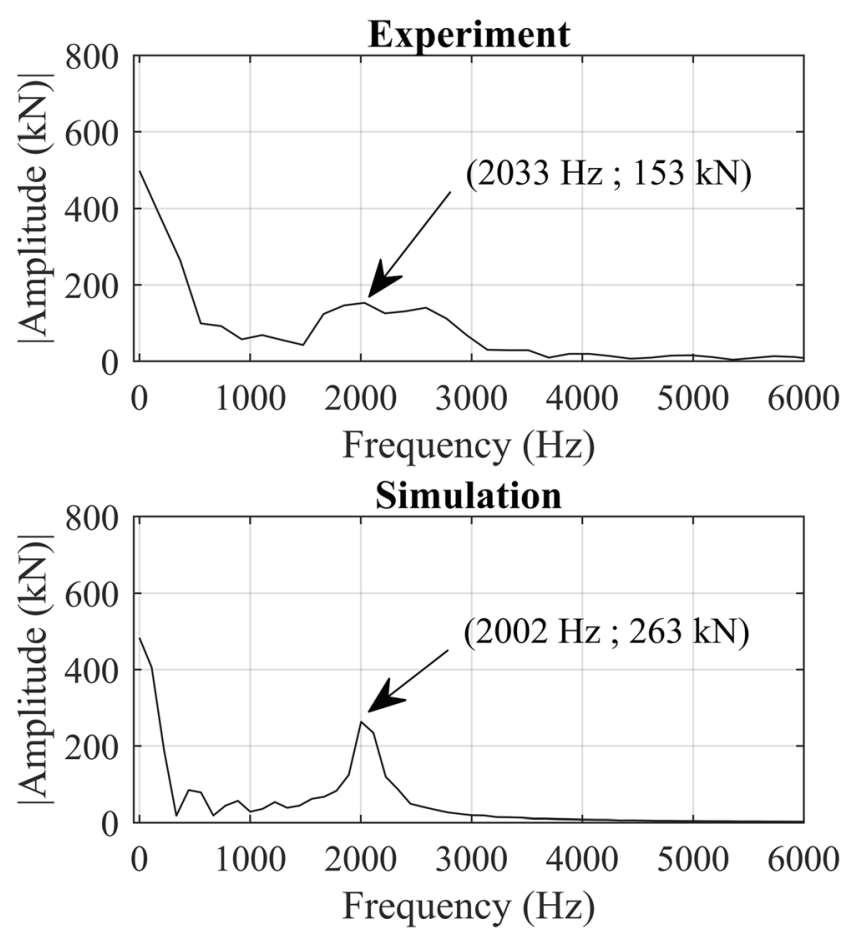

Fig. 11 Frequency spectrums of the experimental and simulated load signals for the 3rd blow on the steel billet 


\subsubsection{Simulation}

The model used to simulate the six blows and the associated model parameters are the same as those presented in section 2.3.1. Only parameters linked to the material are changed and adapted to the aluminium billet. The forging load is calculated according to equation (3). Flow stress is determined for each blow, as presented in section 2.3.2.

The simulations are consecutively performed: Outputs of the simulation $i$ are used as inputs for simulation $i+1$. For each simulated blow, the impact velocity $v_{0}$ and the saturating flow stresses $\sigma_{0}$ are fixed to the measured values. The initial billet height for the simulation of the first blow is fixed to the measured billet height. For the other blows, the initial billet height is fixed to the value of the final billet height from the previous simulation.

\subsubsection{Results}

Figure 12 presents the experimental and the simulated load signals for the 6 blows on the aluminium billet. After each blow, the forging time decreases and the final load increases, both experimentally and numerically. The simulation provides a good prediction of the forging load before reaching the final load. During the spring back, bigger differences can be observed. For the six blows, the second vibratory mode is excited, which produces vibrations on the simulated load signals. Experimentally, these vibrations are noticeable for all the blows, so it is important to consider it within the model.
Fig. 12 Load signals from experiments and simulation for 6 consecutive blows on the aluminium billet

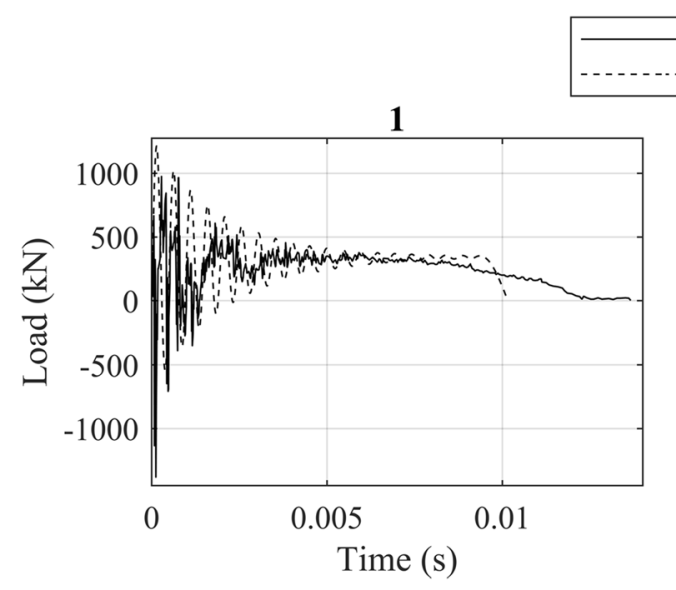

Experimental Simulation
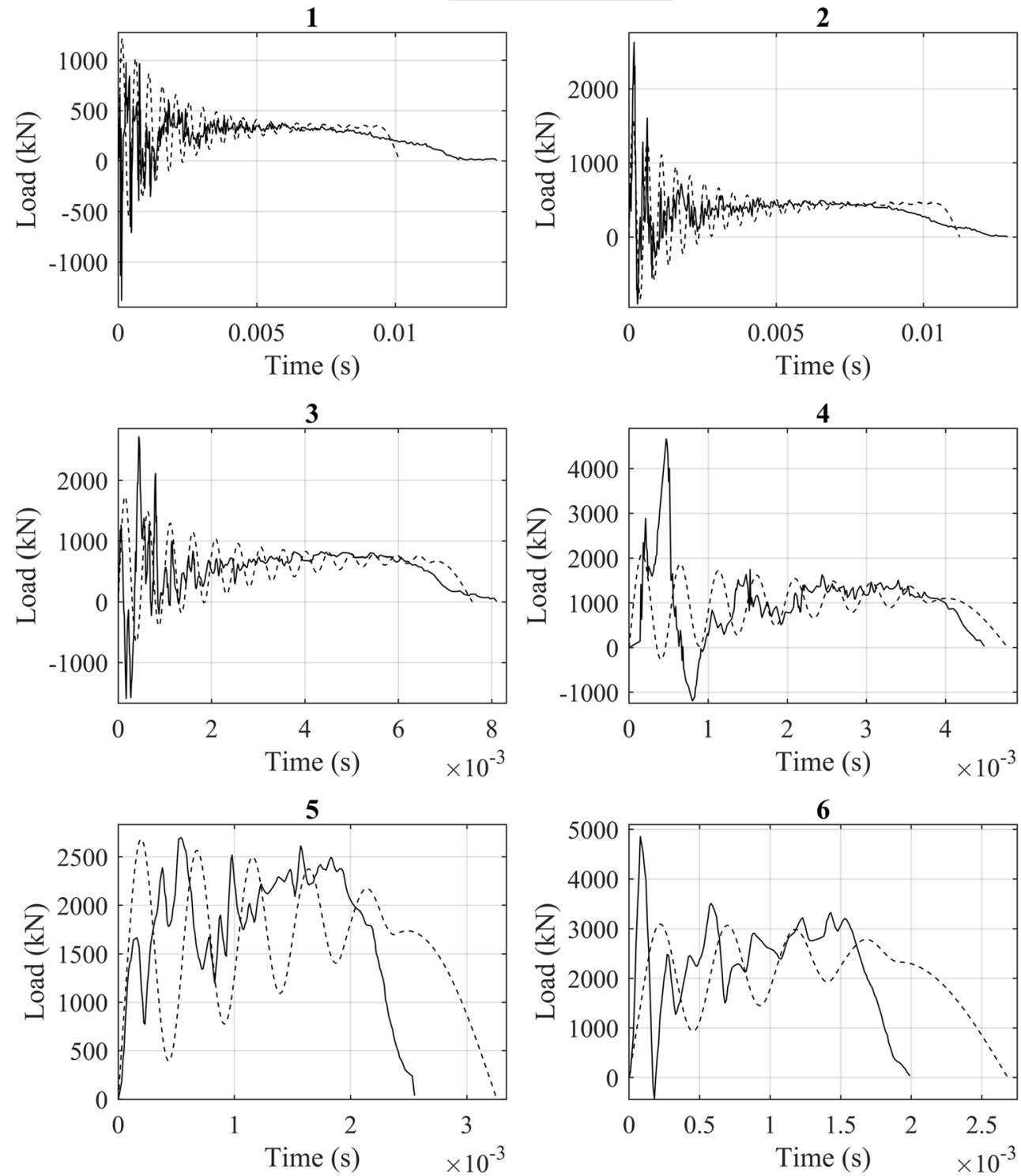

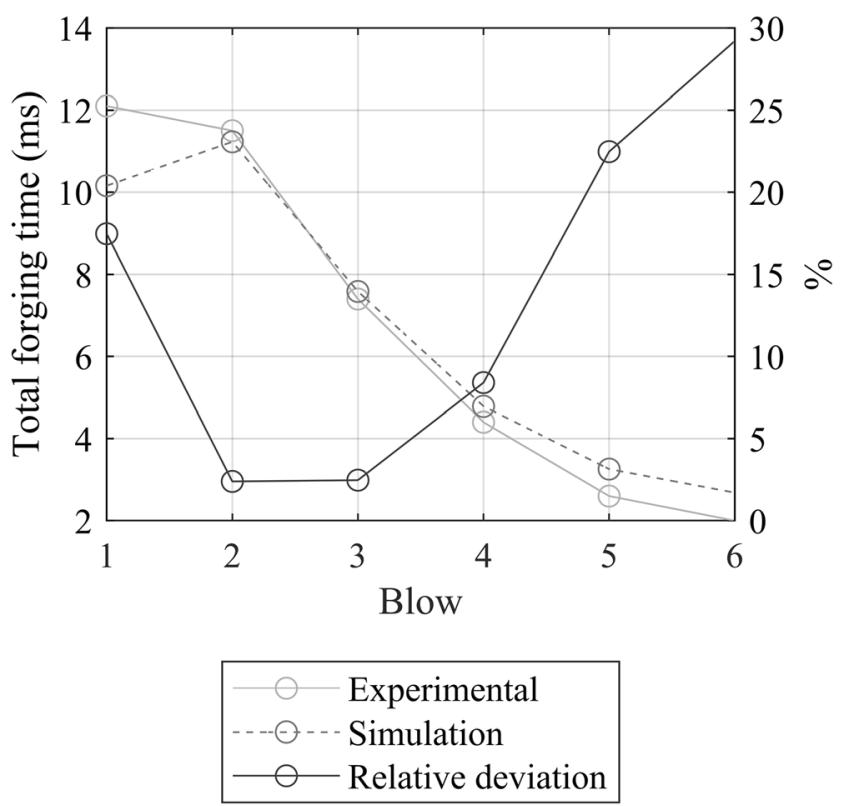

Fig. 13 Measured and simulated forging time for 6 consecutive blows on aluminium billet

The total forging time until the return of the load to zero, the final billet height and the final load are presented in Figs. 13,14 and 15, respectively, in order to compare experimental and simulated load signals. The simulated forging time follows the tendency of the experimental forging time. Except for the first blow, the simulation overestimates the forging time because of the overestimation of spring back time. The increase in the relative deviation with the number of blows can be seen. The relative deviation ranges between 2.4 and $29 \%$
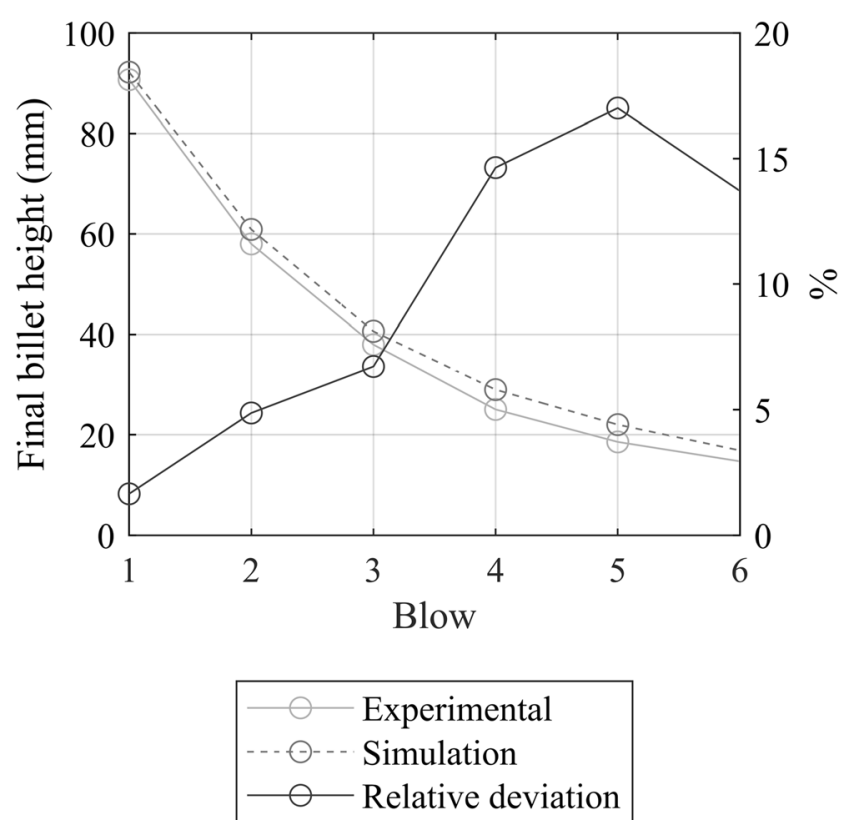

Fig. 14 Measured and simulated billet height for 6 consecutive blows on aluminium billet
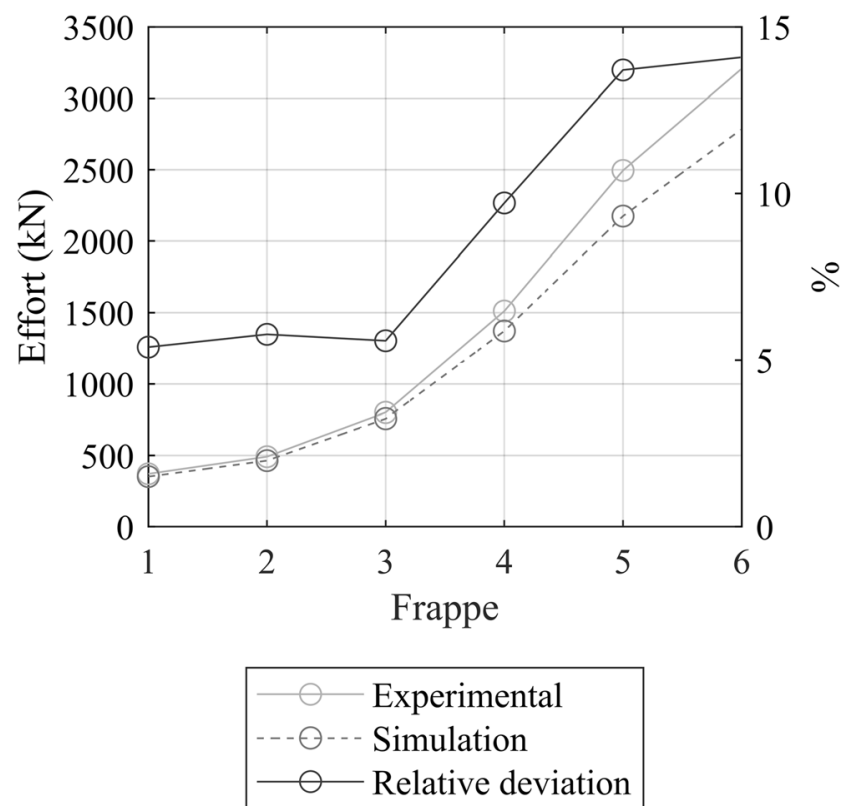

Fig. 15 Measured and simulated final load for 6 consecutive blows on aluminium billet

for a mean value of $13 \%$. For the final billet height, the simulation overestimates the experimental results. Once again, the relative deviation increases with the number of blows, ranging between 2 and $17 \%$. The mean of the relative deviation is equal to $9 \%$. Finally, the simulated final load is lower than the experimental measurement for each blow. The maximum value of the relative deviation is $14 \%$, whereas the lowest value is equal to $5 \%$. The mean of relative deviations is equal to $9 \%$ in the case of the final forging load. It should be noted that the relative error increases with the number of blows for the 3 indicators, which is explained by the accumulation of errors due to the consecutive simulations.

The energy distribution in the BIM system can be determined and then the efficiency of each blow can be calculated. The efficiency is defined as the ratio of the energy transmitted to the billet as plastic energy to the kinetic energy input from the motion of the two masses. The simulated energy distribution at the end of the forging process and the efficiency of each blow are presented in Fig. 16. After the billet forming, the kinetic energy input is turned into four types of energy: the plastic energy transferred to the billet, the energy dissipated in the friction between the billet and the tools, the energy damped in the machine and the kinetic energy of the masses due to their velocity after the spring back. During the forging process, a part of the kinetic energy input is turned into elastic deformation energy in the machine. After reaching the final load, the machine relaxes the elastic deformations and the masses are accelerated in the opposite direction from the stroke. Thus, the kinetic energy of the masses, after the spring back, represents the energy stored in the machine as elastic deformation and not transmitted to the billet. 

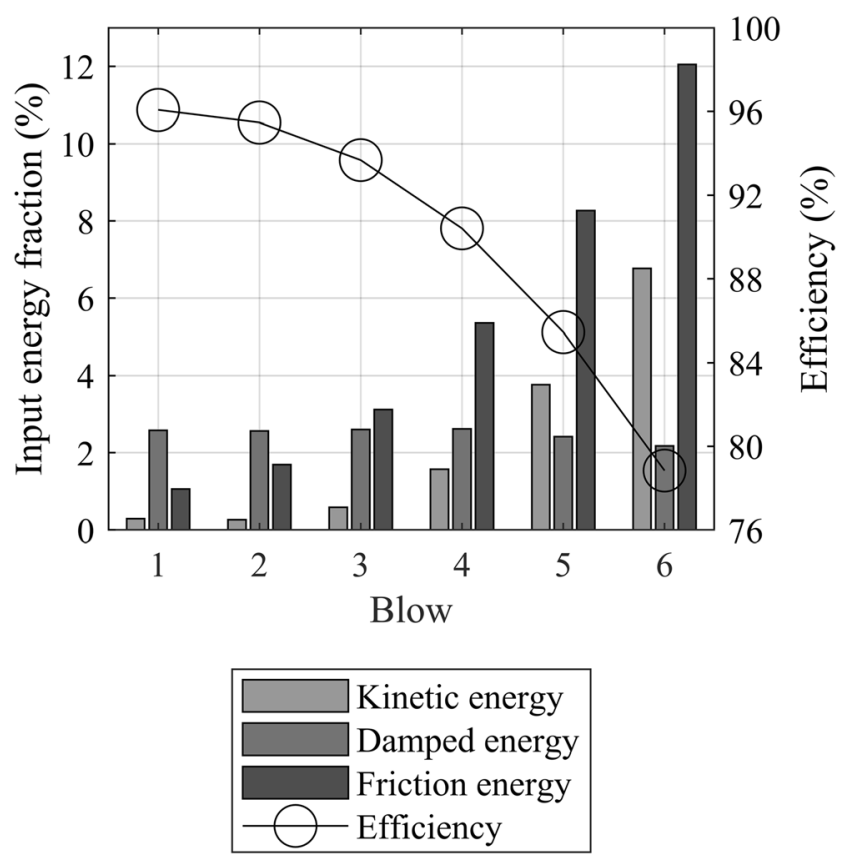

Fig. 16 Simulated efficiency and distribution of the energy at the end of the billet forming for the six blows on the aluminium billet

Efficiency is a decreasing function of the number of blows, which ranges between 96 and $79 \%$ from the first to the sixth blow. Indeed, the higher the number of blows, the more the blow energy is turned into friction and kinetic energy and not into plastic energy. Besides this, the damped energy stays stable whatever the number of blows, and ranges between 2.2 and $2.6 \%$ with no apparent trend.

\section{Discussion}

\subsection{Identification of the model and its parameters}

The protocol presented in this paper provides a new experimental solution to consider machine and tool behaviour during the forming process. With only the measurement of the load signal, the impact velocity, the friction coefficient, and the material characteristics for one blow, it is possible to determine a model and its associated parameters. Dynamic models for forging machines were developed for laboratory drop hammer [7], single-acting hammer [5] and industrial hammers by focusing on foundation behaviour [11] However, model parameters were not determined from experimental measurements and do not consider the specificities of the machine. With new measurement tools, it has become possible to obtain the impact velocity and the load signal during hammer-forming operations. Thus, parameters for a model that considers machine and tool behaviour can be identified from experimental measurements. Because of the experimental identification of the parameters, the model obtained is specifically adapted to the machine-tools system and provides an accurate representation of the forging process, as has been shown by the experimental validation for different materials. In this study, only the forming part of the stroke is considered in the modelling process. The measurement of the impact velocity provides the introduced kinetic energy at the beginning of the blow, thus modelling the acceleration of the mass before the contact with the billet is not needed. Furthermore, the mass rebound has no influence on the useful energy transmitted to the billet, therefore, only focusing on the billet forming is enough to determine the blow efficiency of the forming process.

Two degrees of freedom models are commonly used in the literature to describe hammers $[5,11,15,17]$. These models are good tools for predicting forging variables and blow efficiency, supporting the choice of a two degrees of freedom model in the present study. The model proposed is a simple approximation based on experimental measurements. Model validity is explored with several comparisons between model predictions and experimental measurements. The final objective is to provide a coupling between a machine-tools model and a commercial finite-element model representing billet behaviour. The reasonable number of degrees of freedoms of the model is an asset allowing the coupling possible and limiting computation time. Other studies proposed finite-element models or hybrid models to describe machine behaviour $[35,36]$, but these methods need detailed knowledge about machine structure and important computation time. Thus, the method proposed in this work is an alternative approach, providing a representation that is less sophisticated than a finite-element model of the complete machine-tools system, but is applicable to any hammers, with an acceptable computation time.

An important deviation should be noted in the mass $m_{l}$ that can be compared to the ram-tools mass. Because hammers are not equivalent to a spring-mass-damping system, the model can only be considered as a simplification of machine behaviour. The fitting process provides the parameters, which allow us to reproduce the experimental load signal in simulation, but parameters are not obtained from the geometrical and material characteristics of the structure. This explains the biggest part of the differences between the mass of the hammer and the mass $m_{1}$. Furthermore, the identification of the model parameters is sensitive to the process variables, such as velocity, billet geometries, flow stress, friction coefficient and recorded load. Uncertainties in these variables cause uncertainties in the identified model parameters, which might contribute to the noticed deviation. The latter can also be caused by the assumptions made when modelling rheological material behaviour and friction phenomena. The material flow stress was supposed to be constant and equal to the flow stress at the instant of the final load. Friction coefficient was assumed to be constant during the entire forming process; however, for cylinder upsetting, it has been shown that this coefficient 
increases with the decrease of billet height [37]. Nevertheless, the measurement methods of the process variables and load being the same for the three experimental protocols, it does not affect the proof of relevance of this methodology.

\subsection{Prediction of the first and the third blow for the upsetting of the steel billet}

Simulations of the first and the third blow on the steel billet allow good predictions of the experimental load signal and the billet height (Figs. 8 and 10). It can be noted that, in both cases, the simulated spring back is faster than in the experiments. This may be explained by a change in the element that was experimentally solicited during the spring back, but not considered by the model. Nevertheless, the spring back has no influence on the blow efficiency. The model provides an accurate representation during the forming phase, which is enough for the prediction of the useful energy transmitted to the billet.

\subsection{Prediction of consecutive blows for the upsetting of an aluminium billet}

The model can provide an accurate prediction of the load and the forging indicators for the aluminium billet upsetting. It shows that the parameter identification is not affected by the billet behaviour and it validates the predictive capacity of the model for different forging operations and materials.

The energy that dissipates due to friction increases with the number of blows (Fig. 16) because the area in contact with the tools increases. The kinetic energy shown in Fig. 16 corresponds to the energy stored in the machine structure as elastic deformations and then restituted in the motion of the masses after the billet forming. The increase of the kinetic energy with the number of blows is significant. Since the forging load is higher, blow after blow, and deformations are proportional to the load applied, more energy is stored in the elastic deformations of the machine during the billet forming. Thus, more energy can be restituted during the spring back once the billet forming ended. Concerning damping energy, it stays constant whatever the blow. For each blow, the damped energy is very near to the initial kinetic energy of mass 2 , with a maximum relative deviation for the 4th blow of $6 \%$. Thus, the damped effects are mainly observed for the vibrations of mass 2 . As can be seen in Fig. 12, the vibration of the second vibratory mode is almost completely damped at the end of the billet forming, whatever the blow. This shows that the value of the damped energy is constant, relative to the total kinetic energy input.

Similar results, concerning the evolution of the efficiency in simulation, can be found in the literature. Indeed, Tobias [4] highlights the decrease of efficiency with a decrease in the billet height, for a hammer-forging machine. Blows with small load and large billet deformation are qualified as soft blows and the associated efficiency is high, between 80 and $90 \%$. For hard blows, high load and small billet deformations are noticed and efficiency is low, between 50 and $20 \%$ [38]. In our case, blows 1, 2, 3 and 4 can be considered as soft blows, whereas blows 5 and 6 are intermediary blows, between soft and hard blows. The tendency of the efficiency curve indicates that the following blows would tend towards hard blows. Therefore, it shows that the model can predict the change from soft to hard blow and predicts the useful energy for the billet deformation.

The approach presented in this paper completes the understanding of energy consumption in the hammer-forging process. The method can be applied to existing machines to better quantify the useful energy transmitted to the workpiece. The approach seems suitable for most energy-driven machines, such as hammers or screw presses; further investigations should be conducted to validate the genericity of the method. When impact velocity can be controlled accurately and precisely, as for servo screw presses [39], the BIM model could improve the prediction of the number of blows needed to achieve the final shapes of new workpieces during industrialisation phases. Moreover, knowledge of the actual energy transmitted to the billet will allow better prediction of the thermomechanical path during forging operations and may improve the microstructure control for high-performance materials forging. Process efficiency is influenced by process parameters, such as impact velocity and initial billet geometries [5]. Thanks to the BIM model, these parameters could be optimised, to maximise efficiency for a sequence of forging operations. In this work, billet and interface behaviours were described by analytical models for direct resolution of model equations. This is the first stage that allows the validation of the relevance of a spring-mass-damping model and an experimental identification of its parameters. The next step is to develop a coupling between a finite elements model describing billet and interface behaviour with the spring-massdamping model. Thus, simulation accuracy will be improved and any initial or final shapes for the workpiece might be considered.

\section{Summary and conclusions}

This study presents an experimental approach-modelling hammer-forming operations with a spring-mass-damping vibration model. The degree of freedom and the parameters associated with the model were determined with a stroke on a steel billet. Then, simulations were performed to predict other blows for steel and aluminium billets. Simulated results concerning forging load and final billet height were compared to experimental measurements and showed a good agreement. Finally, the energy distribution predicted by the model was 
analysed. The results are in accordance with observations in the literature. The following conclusions were drawn:

1. The methodology developed to model forming operations on a hammer and identify its associated parameters is relevant. The experimental protocol is near to the working conditions and only requires the measurement of five process parameters, in addition to the forging load signal.

2. The result is a dynamic model that is specifically tailored to the machine-tools system. Simulations realised with this model give an accurate representation of the phenomena that occur during the forging process.

3. The model and its associated parameters, determined for a reference blow on a steel billet, remain valid for aluminium billet upsetting. Parameters are transferable to several forging operations, validating the predictive capacity of the model.

4. The billet-interface-machine model provides a faithful representation of the energy distribution in the system. The model simulates the change from inelastic to intermediate blows and the decrease in the process efficiency with the number of blows. The evolution of efficiency indicates that the model can simulate hard blows. This provides a better understanding of the energy consumption in forging processes and a predictive tool to estimate blow efficiency.

Acknowledgements The authors wish to express their sincere appreciation to Marc Payelleville from the lycée Marie Curie of Nogent-sur-Oise for the realisation of the experimental protocols. Moreover, we would like to thank MANOIR INDUSTRIES and the REGION GRAND EST for their financial support in this research project.

Authors' contributions Not applicable

Funding This research project is supported by MANOIR INDUSTRIES.

Data availability Not applicable

\section{Compliance with ethical standards}

Conflicts of interest The authors declare that they have no conflict(s) of interest.

Code availability Not applicable

\section{References}

1. Durand C, Bigot R, Baudouin C (2018) Contribution to the characterization of metal forming machines: application to screw presses. 17th Int Conf Met Form Met Form 2018 16-19 Sept. 2018

2. Bigot R, Leleu S, Martin P (2003) Forming machine qualification by analysis of manufactured parts geometry: application to aluminium forming process. Int J Adv Manuf Technol 21:476-482. https://doi.org/10.1007/s001700300056
3. Brecher C, Esser M, Witt S (2009) Interaction of manufacturing process and machine tool. CIRP Ann 58:588-607. https://doi.org/ 10.1016/j.cirp.2009.09.005

4. Tobias S (1985) Survey of the development of Petro-Forge forming machines. Int J Mach Tool Des Res 25:105-197

5. Vajpayee S, Sadek MM, Tobias SA (1979) The efficiency and clash load of impact forming machines to the second order of approximation. Int J Mach Tool Des Res 19:237-252. https://doi.org/10. 1016/0020-7357(79)90013-1

6. Vajpayee S, Sadek MM (1977) Analytical study of forming efficiency as influenced by the process and the machine structure. Int $\mathrm{J}$ Prod Res 15:203-218. https://doi.org/10.1080/ 00207547708943118

7. Gregorian V, Sadek MM, Tobias SA (1976) Noise generated by a laboratory drop hammer and its interrelation with the structural dynamics and process parameters. Int J Mach Tool Des Res 16: 301-318. https://doi.org/10.1016/0020-7357(76)90040-8

8. Vajpayee S, Sadek MM, Tobias SA (1982) Prediction of linear noise-load relationship for impact forming machines. Int J Mach Tool Des Res 22:1-6. https://doi.org/10.1016/0020-7357(82) 90015-4

9. Vajpayee S, Sadek MM (1978) Effects of structural and forming parameters on the efficiency of energy transfer in impact forming machines. J Eng Ind 100:113-118. https://doi.org/10.1115/1. 3439397

10. Novak M (1983) Foundations for shock-producing machines. Can Geotech J 20:141-158. https://doi.org/10.1139/t83-013

11. Novak M, El Hifnawy L (1983) Vibration of hammer foundations. Int J Soil Dyn Earthq Eng 2:43-53. https://doi.org/10.1016/02617277(83)90026-8

12. El Hifnawy L, Novak M (1984) Response of hammer foundations to pulse loading. Int J Soil Dyn Earthq Eng 3:124-132. https://doi. org/10.1016/0261-7277(84)90042-1

13. Harwood M, Novak M (1986) Uplift in hammer foundations. Soil Dyn Earthq Eng 5:102-117. https://doi.org/10.1016/02677261(86)90004-7

14. Chow YK, Yong DM (1993) Hammer foundation analysis by the wave equation. Comput Struct 47:107-110. https://doi.org/10. 1016/0045-7949(93)90283-J

15. Chehab AG, El Naggar MH (2003) Design of efficient base isolation for hammers and presses. Soil Dyn Earthq Eng 23:127-141. https://doi.org/10.1016/S0267-7261(02)00157-4

16. Chehab AG, El Naggar MH (2004) Response of block foundations to impact loads. J Sound Vib 276:293-310. https://doi.org/10.1016/ j.jsv.2003.07.028

17. Wang G, Dong Z (2006) Design optimization of low impact transmission foundation for forging hammers. Eng Comput 23:166-186. https://doi.org/10.1108/02644400610644531

18. Heidari M, El Naggar MH (2010) Using reinforced soil systems in hammer foundations. Proc Inst Civ Eng - Ground Improv 163:121132. https://doi.org/10.1680/grim.2010.163.2.121

19. Dindorf R, Wos P (2020) Energy-saving hot open die forging process of heavy steel forgings on an industrial hydraulic forging press. Energies 13:1620

20. Jomartov A, Tuleshov A, Kuatova M (2020) Dynamic model of a crank press in the process of braking. In: Kuo C-H, Lin P-C, Essomba T, Chen G-C (eds) Robotics and Mechatronics. Springer International Publishing, Cham, pp 141-150

21. Swidergal K, Lubeseder C, von Wurmb I, Lipp A, Meinhardt J, Wagner M, Marburg S (2015) Experimental and numerical investigation of blankholder's vibration in a forming tool: a coupled MBS-FEM approach. Prod Eng 9:623-634. https://doi.org/10. 1007/s11740-015-0640-9

22. Zheng E, Zhou X (2014) Modeling and simulation of flexible slider-crank mechanism with clearance for a closed high speed 
press system. Mech Mach Theory 74:10-30. https://doi.org/10. 1016/j.mechmachtheory.2013.11.015

23. Brecher C, Klein W, Tannert M (2010) Optimization of multi-stage closed-die forging processes by coupled simulation of the machine and the forging processes. Prod Eng 4:279-286. https://doi.org/10. 1007/s11740-010-0226-5

24. Hongtang L, Bufang L (2003) Research of the dynamic characteristics on a new hydraulic system of electro-hydraulic hammer. Chin J Mech Eng 1:24-26

25. Zhang YJ (2013) Research of all-hydraulic die forging hammer control system. Adv Mater Res 785:1286-1289

26. Zhu M, Liang J, Zhao S (2018) Designing of an electromagnetic driver for the reversing valve of CNC die forging hammer. Proc Inst Mech Eng Part C J Mech Eng Sci 232:358-368

27. Galdos L, de Argandoña ES, Herrero N et al (2014) The calibration of high energy-rate impact forging hammers by the copper-column upsetting method and high speed Camera measurements. Key Eng Mater 611-612:173-177. https://doi.org/10.4028/www.scientific. net/KEM.611-612.173

28. Chen R, Li Z, Zhong K, Liu X, Wu Y, Wang C, Shi Y (2019) A stereo-vision system for measuring the ram speed of steam hammers in an environment with a large field of view and strong vibrations. Sensors 19:996. https://doi.org/10.3390/s19050996

29. Yoneyama T (2017) Development of a friction sensor for hot forging. Int J Adv Manuf Technol 90:2251-2261. https://doi.org/10. 1007/s00170-016-9539-7

30. Yoneyama T, Miyoshi K, Tate T (2018) Contact stress and temperature during air-stamp hammer upsetting of a circular cylinder. $\mathrm{J}$ Manuf Process 32:791-801. https://doi.org/10.1016/j.jmapro.2018. 04.012

31. Borkowski J, Mroczka J (2010) LIDFT method with classic data windows and zero padding in multifrequency signal analysis.
Measurement 43:1595-1602. https://doi.org/10.1016/j. measurement.2010.09.001

32. Lange K (1985) Handbook of metal forming. McGraw-Hill Book Co 1985:1216

33. Szeliga D, Gawad J, Pietrzyk M (2006) Inverse analysis for identification of rheological and friction models in metal forming. Comput Methods Appl Mech Eng 195:6778-6798. https://doi. org/10.1016/j.cma.2005.03.015

34. Metropolis N, Ulam S (1949) The Monte Carlo method. J Am Stat Assoc 44:335-341

35. Swidergal K, Lubeseder C, von Wurmb I, Marburg S (2014) Vibration analysis of an automotive forming tool using coupled MBS-FEM simulation and experimental validation. Conf 26th Int Conf Noise Vib Eng ISMA2014

36. Zheng EL, Jia F, Zhang ZS, Shi JF (2011) Modeling and simulation of nonlinear combination disc-spring vibration isolator for highspeed press. Adv Mater Res 211-212:40-47. https://doi.org/10. 4028/www.scientific.net/AMR.211-212.40

37. Li YP, Onodera E, Matsumoto H, Chiba A (2009) Correcting the stress-strain curve in hot compression process to high strain level. Metall Mater Trans A 40:982-990. https://doi.org/10.1007/s11661009-9783-7

38. Altan T, Ngaile G, Shen G (2005) Cold and hot forging: fundamentals and applications. ASM International

39. Halicioglu R, Dulger LC, Bozdana AT (2016) Mechanisms, classifications, and applications of servo presses: a review with comparisons. Proc Inst Mech Eng Part B J Eng Manuf 230:1177-1194. https://doi.org/10.1177/0954405415600013

Publisher's note Springer Nature remains neutral with regard to jurisdictional claims in published maps and institutional affiliations. 